03.1

\title{
Отрицательное маховское отражение с множественными тройными конфигурациями при дифракции ударной волны на клине
}

\author{
(ㄱ П.Ю. Георгиевский, А.Н. Максимов, В.П. Фокеев
}

Научно-исследовательский институт механики Московского государственного университета им. М.В. Ломоносова, Москва, Россия

E-mail: georgi@imec.msu.ru

Поступило в Редакцию 28 мая 2021 г.

В окончательной редакции 2 июля 2021 r.

Принято к публикации 5 июля 2021 г.

В рамках уравнений Эйлера выполнено численное исследование структуры автомодельного течения для различных типов отрицательного маховского отражения при дифракции ударной волны на клине. Наряду с известными режимами двойного и тройного маховского отражения обнаружен качественно новый режим отрицательного маховского отражения с множественными тройными конфигурациями. Отмечены особенности перехода от режима отражения с множественными тройными конфигурациями к регулярному отражению при изменении угла клина.

Ключевые слова: ударная волна, тройная конфигурация, двойное маховское отражение, отрицательное маховское отражение.

DOI: 10.21883/PJTF.2021.20.51606.18889

Задача о дифракции ударной волны на клине в классической постановке (для идеального совершенного газа с постоянным показателем адиабаты) характеризуется удивительным многообразием автомодельных решений, что обусловливает неизменный интерес к ней классиков и современных исследователей - специалистов в области газовой динамики и физики ударных волн. Обзор актуальных и нерешенных проблем и современная классификация режимов маховского отражения ударных волн приведены в [1-3].

При отрицательном двойном маховском отражении в первой тройной конфигурации падающая и отраженная ударные волны располагаются по разные стороны от прямолинейной траектории движения тройной точки. Отрицательное двойное маховское отражение для умеренных значений числа Маха падающей ударной волны реализуется в газах с показателем адиабаты, близким к единице (в экспериментах для тяжелых газов со сложной молекулярной структурой). Экспериментально отрицательное двойное маховское отражение в задаче о дифракции ударной волны на клине обнаружено в $[4,5]$. Условия реализации различных режимов двойного маховского отражения (положительного и отрицательного) обсуждались в [6-8]. В [9] исследованы эффекты формирования тонких возвратных струй вблизи поверхности клина и интенсивных циркуляционных течений за маховской ножкой при двойном маховском отражении. Аналитическое исследование областей существования стационарных тройных конфигураций с отрицательным отражением проведено в $[10,11]$.

В настоящей работе выполнено численное исследование структуры автомодельного течения для различ- ных типов отрицательного маховского отражения при дифракции ударной волны на клине.

Для описания плоских нестационарных течений совершенного идеального газа в декартовой системе координат $(x, y)$ использовались уравнения Эйлера

$$
\frac{\partial}{\partial t}\left(\begin{array}{c}
\rho \\
\rho u \\
\rho v \\
e
\end{array}\right)+\frac{\partial}{\partial x}\left(\begin{array}{c}
\rho u \\
\rho u^{2}+p \\
\rho u v \\
(e+p) u
\end{array}\right)+\frac{\partial}{\partial y}\left(\begin{array}{c}
\rho v \\
\rho u v \\
\rho v^{2}+p \\
(e+p) v
\end{array}\right)=0
$$

где $p$ - давление, $\rho$ - плотность, $u, v-$ компоненты скорости вдоль осей $x, y$ соответственно, $e-$ полная энергия единицы объема, которая для совершенного газа с постоянным показателем адиабаты $\gamma$ имеет вид $e=p /(\gamma-1)+\rho\left(u^{2}+v^{2}\right) / 2$. Задача приведена к безразмерному виду, давление и плотность покоящегося газа перед ударной волной используются в качестве масштабов.

Расчетная область выбиралась таким образом, чтобы возмущения не доходили до ее границ (рис. 1). Ось абсцисс прямоугольной декартовой расчетной сетки совпадает с поверхностью клина. По заданному числу Маха $\mathrm{M}_{s}$ падающей ударной волны из соотношений Ренкина-Гюгонио вычисляются параметры потока $u_{s}$, $v_{s}, \rho_{s}, p_{s}$ за ней. В начальный момент времени ударная волна проходит через вершину клина $x_{0}=1 / 6$ и наклонена под углом $90^{\circ}-\alpha(\alpha-$ угол клина $)$. В расчетных точках слева от нее параметры потока соответствуют течению за ударной волной, а справа - состоянию покоя перед ней. Расчет течения проводился в области $0 \leqslant x \leqslant 4,0 \leqslant y \leqslant 1$. Поскольку точка пересечения падающей ударной волны с верхней границей расчетной области в любой момент времени известна, можно 


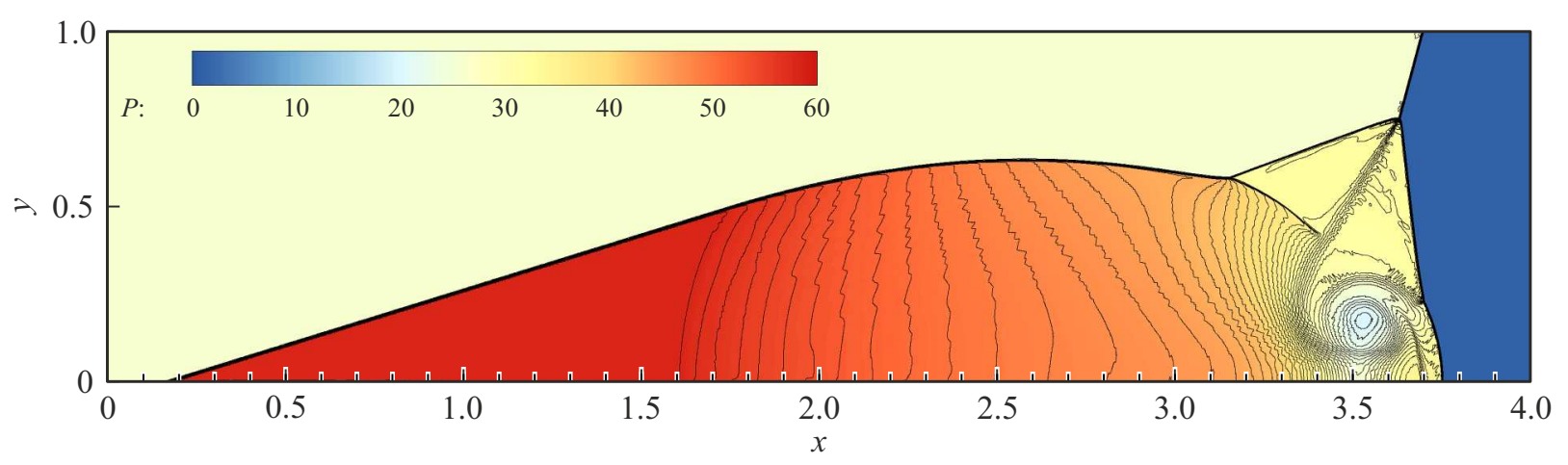

Рис. 1. Расчетная область и тестовый расчет для случая отрицательного „тройного“ отражения Маха-Уайта. Поле давления показано цветом, плотности - контурами (цветной вариант рисунка представлен в электронной версии статьи). $\mathrm{M}_{s}=4.94$, $\gamma=1.13, \alpha=15^{\circ}$.

задать точные условия на верхней, левой (входной) и правой (выходной) границах. На нижней границе на отрезке $0 \leqslant x \leqslant x_{0}$ задавались условия свободного вытекания, а на отрезке $x_{0} \leqslant x \leqslant 4$ (поверхности клина) условия непротекания (реализованы как условия симметрии для $p, \rho, u$ и отражения для $v$ ). Безразмерными определяющими параметрами задачи являются число Маха падающей ударной волны $\mathrm{M}_{s}$, показатель адиабаты газа $\gamma$ и угол клина $\alpha$. Корректно рассчитанное решение задачи должно быть стационарным в автомодельных переменных $\xi=\left(x-x_{0}\right) / t, \eta=y / t$.

Численное исследование выполнено с использованием оригинальной программы, в которой реализована TVD-модификация [12] явной конечно-разностной схемы МакКормака [13], обеспечивающая сохранение монотонности решения. Как показало сравнительное тестирование на различных одномерных и двумерных задачах современной газовой динамики, выполненное авторами по методике [14], данная схема обеспечивает надежный расчет нестационарных течений с газодинамическими разрывами различного типа на подробных сетках. Стандартное разрешение расчетной сетки составляло $6400 \times 1600$, а в специально оговоренных случаях использовалась сетка удвоенного разрешения $12800 \times 3200$. Далее во всех расчетах некоторые параметры задачи зафиксированы: $\mathrm{M}_{s}=4.94, \quad \gamma=1.13$ $\left(\mathrm{CCl}_{2} \mathrm{~F}_{2}\right.$ - Freon R12).

Для задачи о дифракции ударной волны на клине проведена верификация в соответствии с методикой [15], а также выполнено сравнение с экспериментальными и расчетными данными [3] для различных режимов маховского отражения. На рис. 1 показан случай отрицательного „тройного“ отражения Маха-Уайта (triple Mach-White reflection), реализующийся при $\alpha=15^{\circ}$, соответствующий эксперименту [3] (см. рис. 1, $h$ в указанной работе). Цветом показано распределение давления, а контурами - распределение плотности, что позволяет визуально различать ударные волны и тангенциальные разрывы (цветной вариант рисунка представлен в электронной версии статьи). Основными особенностями течения являются интенсивное циркуляционное течение под тангенциальным разрывом, мощная возвратная струя вдоль поверхности и наличие дополнительной тройной точки на маховской ножке (что позволяет классифицировать данный случай как „тройное“ отражение).

В настоящей работе обнаружены и исследованы качественно новые автомодельные режимы отрицательного маховского отражения с множественными тройными конфигурациями, что позволяет классифицировать их как „многократное“ маховское отражение. На рис. 2 показаны поля течения при $\alpha=35^{\circ}$ (разрешение расчетной сетки $12800 \times 3200)$. Векторами отображается „автомодельное“ поле скоростей в соответствии с методикой [9]. Для вычисления „автомодельных“ компонент скорости $(U, V)$ в каждой расчетной точке из физического вектора скорости вычитается вектор „автомодельного“ расширения (модуль которого равен расстоянию до вершины клина, деленному на время): $U=u-\xi=u-\left(x-x_{0}\right) / t, V=v-\eta=v-y / t$. „Автомодельное“ поле скоростей в окрестности любой характерной точки течения (например, тройной точки) локально совпадает с физическим полем скоростей в системе отсчета, связанной с этой точкой. Для проверки автомодельности решения проведено сравнение распределений параметров в различные моменты времени, которое подтвердило масштабируемость результатов. Кроме того, были построены теневые картины для поля абсолютных максимумов давления в каждом узле сетки за все время расчета (аналогичная техника применяется для визуализации ячеистой структуры газовой детонации), анализ которых подтвердил прямолинейность траекторий движения тройных точек.

На рис. 2 показаны распределения локальных чисел Маха в „автомодельном“ поле скоростей и относительной температуры $(p / \rho)$, которые позволяют провести анализ ударно-волновых конфигураций и струйных течений. В первой тройной конфигурации 1 угол отражения падающей ударной волны $I S$ является отрицательным, что с необходимостью приводит к появлению на отраженном скачке $R S 1$ второй тройной конфигурации 2 

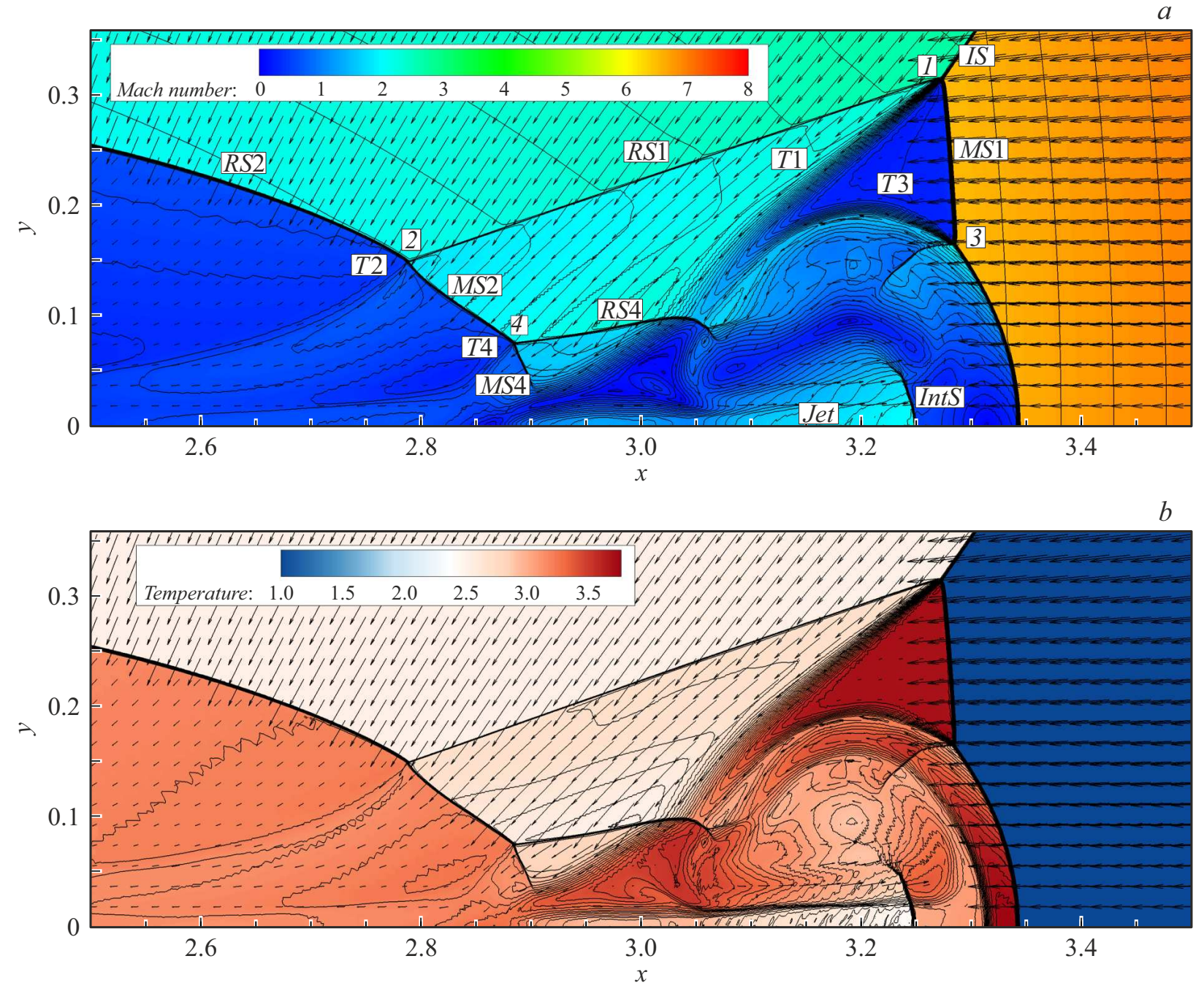

Pис. 2. Структура течения при отрицательном маховском отражении с множественными тройными конфигурациями. $\mathrm{M}_{s}=4.94$, $\gamma=1.13, \alpha=35^{\circ} . a$ - распределение чисел Маха в „автомодельном“ поле скоростей; $b$ - поле температуры. Пояснения в тексте.

со скачками $R S 2, M S 2$ и тангенциальным разрывом T2. При этом течение в обширной „верхней“ области над тангенциальным разрывом $T 1$ является сверхзвуковым. В „нижней“ области под тангенциальным разрывом $T 1$ за маховской ножкой $M S 1$ формируется интенсивное циркуляционное течение, что приводит к появлению третьей тройной конфигурации 3 на $M S 1$. Можно выделить сверхзвуковую возвратную струю Jet, распространяющуюся вдоль поверхности по направлению к $M S 1$, торможение которой осуществляется во внутреннем скачке Int $S$. Установлено, что возникновение новых тройных конфигураций над тангенциальным разрывом $T 1$ связано с интенсификацией циркуляционных струйных течений при увеличении угла клина. В результате взаимодействия внутренней ударной волны $M S 2$ со струей высокотемпературного газа, истекающего из треугольной области, ограниченной скачком $M S 1$ и тангенциальными разрывами $T 1, T 3$, формируется тройная конфигурация 4, включающая косые скачки
$R S 4, M S 4$. Данный результат хорошо согласуется с эффектом формирования крупномасштабного газодинамического предвестника при взаимодействии ударной волны с тонким слоем нагретого газа [16].

Автомодельное течение при многократном маховском отражении устроено так, что значительная часть потока проходит в узкое „горло“ вблизи поверхности клина через скачки $M S 2, M S 4$. При этом около поверхности клина появляется область растекания струй, направленных вдоль поверхности клина к его вершине и от нее, в сторону основной маховской ножки $M S 1$. Давление и плотность в области растекания за скачком $M S 4$ оказываются очень высокими (в расчетах давление в 150 раз превышало начальное). Однако температура (рис. $2, b)$ в области растекания остается умеренной (в 3 раза больше начальной), что меньше, чем температура непосредственно за маховской ножкой $M S 1$. Таким образом, высокотемпературные эффекты реального газа не должны оказывать существенного влияния на решение 

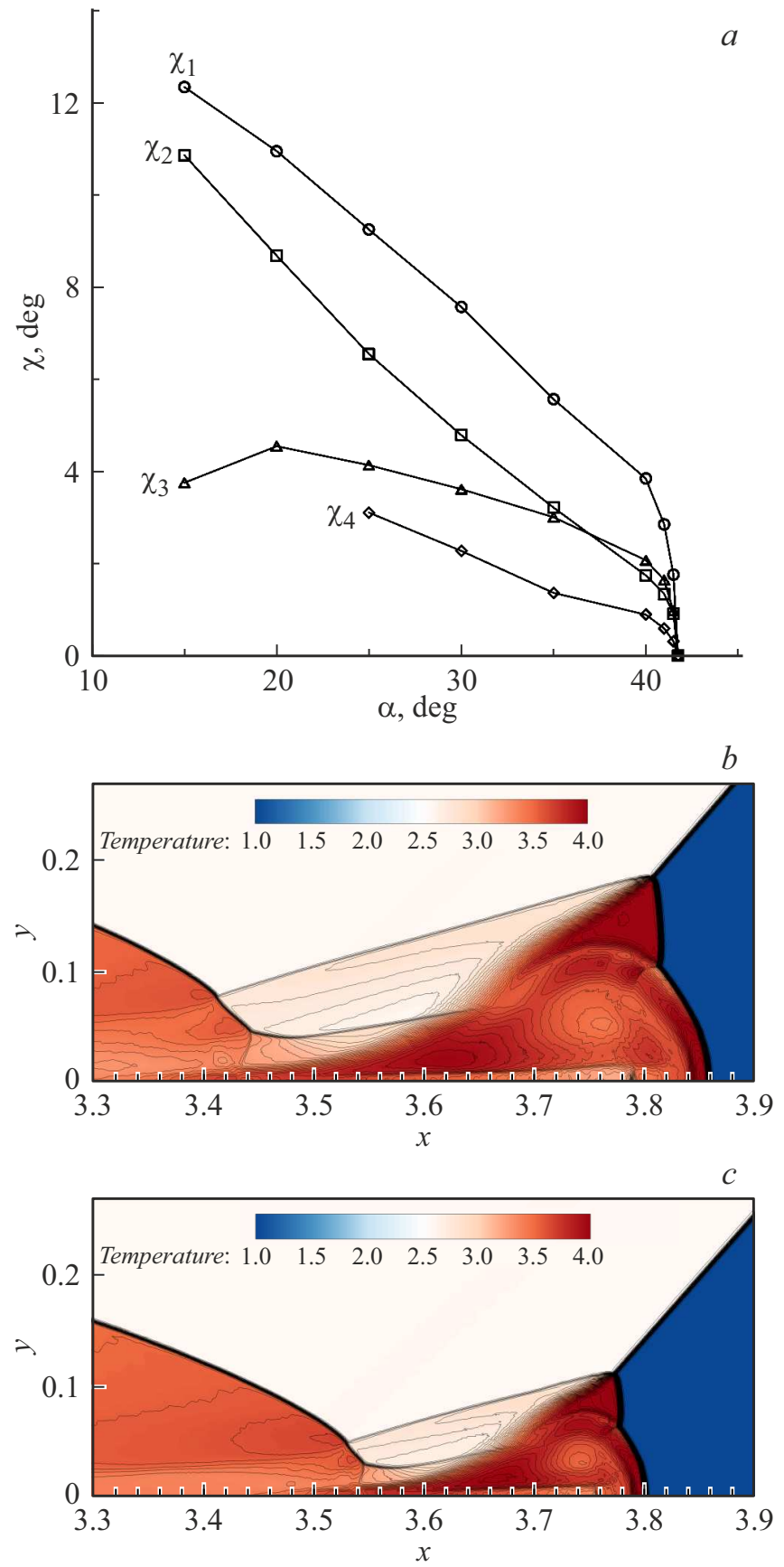

Рис. 3. Особенности перехода к регулярному отражению при изменении угла клина $\alpha$ при $\mathrm{M}_{s}=4.94, \gamma=1.13$. $a-$ угол наклона траекторий тройных точек $\chi$ в зависимости от $\alpha$; $b$ и $c-$ поле температуры при $\alpha=41$ и $41.5^{\circ}$ соответственно.

задачи, и применение модели идеального газа с постоянными теплоемкостями оправдано.

Отмечены особенности эволюции режимов отрицательного маховского отражения при изменении угла клина $\alpha$ (рис. 3). Показано, что при увеличении угла клина последовательно реализуются режимы двойного, тройного и многократного маховского отражения, а затем осуществляется переход к регулярному отра- жению. Установлено, что при увеличении угла клина режим многократного отражения реализуется начиная с угла $\alpha=25^{\circ}$ и сохраняется вплоть до перехода к регулярному отражению при $\alpha=41.75^{\circ}$. При этом на завершающей стадии переход происходит резко, так что зависимость углов наклона прямолинейных траекторий движения тройных точек $\chi$ от угла клина $\alpha$ имеет вертикальные касательные в точке перехода (рис. 3,a). Кроме того, отмечено подобие газодинамических структур при приближении к критическому углу перехода, когда при небольшом изменении угла клина $\alpha$ значительно меняется размер области взаимодействия, но сохраняется масштабируемость течения (рис. $3, b, c)$. При этом качественно картина течения для углов, близких к критическому углу перехода, соответствует экспериментальным данным (см. рис. 2, $a$ в [6] и рис. 7, $d$ в [8]).

Полученное решение с множественными тройными конфигурациями (режим многократного отражения) расширяет представления о возможных газодинамических конфигурациях в задаче о дифракции ударной волны на клине и дополняет современную классификацию [1-3]. Для определения критериев реализации обнаруженных режимов отрицательного маховского отражения и уточнения общих закономерностей перехода к регулярному отражению необходимы дальнейшие исследования.

\section{Финансирование работы}

Работа выполнена в НИИ механики Московского государственного университета им. М.В. Ломоносова при финансовой поддержке Российского научного фонда (проект № 21-11-00307).

\section{Конфликт интересов}

Авторы заявляют, что у них нет конфликта интересов.

\section{Список литературы}

[1] G. Ben-Dor, Shock Waves, 15, 277 (2006). DOI: $10.1007 / \mathrm{s} 00193-006-0036-\mathrm{Z}$

[2] А.Н. Семенов, М.К. Березкина, И.В. Красовская, ЖТФ, 79 (4), 46 (2009). https://journals.ioffe.ru/articles/9658

[A.N. Semenov, M.K. Berezkina, I.V. Krasovskaya, Tech. Phys., 54(4), 491 (2009).

DOI: $10.1134 / \mathrm{S} 1063784209040082]$.

[3] А.Н. Семенов, М.К. Березкина, И.В. Красовская, ЖТФ, 79 (4), 52 (2009). https://journals.ioffe.ru/articles/9659

[A.N. Semenov, M.K. Berezkina, I.V. Krasovskaya, Tech. Phys., 54 (4), 497 (2009).

DOI: $10.1134 / \mathrm{S} 1063784209040094]$.

[4] Л.Г. Гвоздева, О.А. Предводителева, ДАН СССР, 163 (5), 1088 (1965). http://mi.mathnet.ru/dan31451 [L.G. Gvozdeva, O.A. Predvoditeleva, Sov. Phys. Dok1., 10, 694 (1966).]. 
[5] L.G. Gvozdeva, T.V. Bazhenova, O.A. Predvoditeleva, V.P. Fokeev, Astron. Acta, 14, 503 (1969). https://elibrary.ru/item.asp?id=30895396; https://www.scopus.com/record/display.uri?eid=2-s2.0-00145 20333\&origin $=$ resultslist

[6] T.V. Bazhenova, V.P. Fokeev, L.G. Gvozdeva, Acta Astron., 3, 131 (1976). DOI: 10.1016/0094-5765(76)90098-9

[7] J.H. Lee, I.I. Glass, Prog. Aerospace Sci., 21, 33 (1984). DOI: $10.1016 / 0376-0421(84) 90003-4$

[8] L. Gvozdeva, S. Gavrenkov, A. Nesterov, Shock Waves, 25, 283 (2015). DOI: 10.1007/s00193-015-0568-1

[9] L.F. Henderson, E.I. Vasilev, G. Ben-Dor, T. Elperin, J. Fluid Mech., 479, 259 (2003). DOI: 10.1017/S0022112002003518

[10] М.В. Сильников, М.В. Чернышов, Л.Г. Гвоздева, ЖТФ, 86 (11), 30 (2016). DOI: 10.21883/jtf.2016.11.43810.1720 [M.V. Sil'nikov, M.V. Chernyshov, L.G. Gvozdeva, Tech. Phys., 61 (11), 1633 (2016). DOI: 10.1134/S1063784216110232].

[11] M.V. Chernyshov, A.S. Kapralova, K.E. Savelova, Acta Astron., 179, 382 (2021). DOI: 10.1016/j.actaastro.2020.11.015

[12] S.F. Davis, SIAM J. Sci. Stat. Comput., 8, 1 (1987). DOI: $10.1137 / 0908002$; S.F. Davis, in Upwind and High-Resolution Schemes (Springer, 1997), p. 149-166. DOI: 10.1007/978-3-642-60543-7_9

[13] R.W. MacCormack, in 4th Aerodynamic Testing Conf. (Cincinnati, USA, 1969), AIAA Paper 1969-354. DOI: 10.2514/6.1969-354; R.W. MacCormack, J. Spacecraft Rockets, 40, 757 (2003). DOI: 10.2514/2.6901

[14] R. Liska, B. Wendroff, SIAM J. Sci. Comput., 25, 995 (2003). DOI: $10.1137 / \mathrm{S} 1064827502402120$

[15] K. Takayama, Z. Jiang, Shock Waves, 7, 191 (1997). DOI: $10.1007 / \mathrm{s} 001930050075$

[16] П.Ю. Георгиевский, В.А. Левин, О.Г. Сутырин, Письма в ЖТФ, 44 (20), 5 (2018).

DOI: $10.21883 /$ PJTF.2018.20.46887.17402

[P.Yu. Georgievskiy, V.A. Levin, O.G. Sutyrin, Tech. Phys. Lett., 44 (10), 905 (2018).

DOI: $10.1134 / \mathrm{S} 1063785018100231$ 